\title{
Abstract Author Index by abstract number
}

\section{A}

Adermann, P., 16

Aguiar-Ricardo, A., 25

Akhuemokhan, P., 79

Akkerman, O.W., 71

Alffenaar, J.-W.C., 71

Amighi, K., 22

Andersson, P., 69

Angulo, J., 85

Arora, S., 81

Arthur, K., 29

Baker, D., 41

Balducci, A.G., 73, 83

Bansal, H.S., 46

Baron, C., 59

Belotti, S., 40

Besenhard, M.O., 32

Bettini, R., 73, 83

Bimbo, L.M., 17

Birk, G., 06

Biserni, S., 32, 36

Blatchford, C., 68

Bloxham, M., 58

Borella, F., 73

Börjel, M., 51

Bosquillon, C., 27

Brambilla, G., 35, 38, 84

Bresciani, M., 12, 47, 55, 60

Brogin, G., 55

Brouet, G., 59

Brown, A., 40

Brown, M., 54, 57

Bruin, R., 23, 26

Bryan, S., 40

Buckton, G., 58

Buttini, F., 73, 83

C

Cabral-Marques, H.M., 07

Calzolari, V., 32, 36

Campos, S., 03, 25

Chau, D., 57

Chen, Y., 44

Childerhouse, N., 15

Church, T., 35, 38, 84

Cingolani, E., 27

Clarke, G.W., 50, 74

Clarke, J., 75

Colombo, P., 73

Cooper, A., 19

Copley, M., 68
Costa, E., 03, 25

Courtney, S., 76

Cox, B., 85

D

Dailey, L.A., 24

Davidson, N., 80

Dawood, A., 13

de Boer, A.H., 01, 45, 71, 72

Deleers, M., 22

Diez, F., 09, 22

Dogru, M., 05

Dunster, P., 29

Dutton, B., 66

Dzidic, N., 55

$\mathbf{E}$

Edwards, M., 40

Elsayed, M.M.A., 33

F

Farina, D.J., 63

Faulhammer, E., 30, 31, 32, 36, 37

Fernandes, J.V., 08, 70

Ferrari, A., 32

Fisher, B., 70

Fisher, H., 70

Folestad, S., 69

Forbes, B., 24, 54, 66, 74, 79, 83

Forbes, B.F., 50

Fröhlich, E., 12, 55, 60

Frijlink, H.W., 45, 71, 72

Fussell, A.L., 72

G

Gavtash, B., 35, 38, 84

Gerde, P., 51

Ghadiri, M., 34

Giorgetti, M., 85

Govoni, M., 55

Grasmeijer, F., 45, 72

Grosjean, B., 59

\section{H}

Hagedoorn, P., 45, 71

Haghi, M., 81

Hammond, M., 14, 79

Hardaker, L.E.A., 48, 53

Hargrave, G., 35, 38, 84

Hassoun, M., 79

Haswell, S., 78
Hatley, R.H.M., 48, 53

Hebbink, G., 46

Hennia, I., 22

Hertel, M., 05

Hilal, M., 13

Hirvonen, J., 17

Hoffman, E., 41

Hoppentocht, M., 71

Hutcheon, G., 13

Hutter, V., 41, 57

I

Imming, P., 24

Inoue, K., 21

Issa, K.B., 41

Ito, A., 21

J

Jackson, E., 43

Jain, S., 81

Jarolimek, W., 34

Jones, S., 66

Joost, B., 56

Josefson, M., 69

$\mathbf{K}$

Kalberer, M., 80

Kamplade, J., 82

Kanabar, V., 54

Kappl, M., 81

Karlsson, L., 69

Kauppinen, E.I., 17

Keegstra, J.R., 44

Kennedy, S., 43

Kerstjens, H.A.M., 71

Khinast, J., 02, 12, 60

Khinast, J.G., 30, 31, 32, 36, 37, 47

Kopsch, T., 18

Kovalcik, A., 37

Krüger, P., 67

Kuimova, M., 80

Kumar, A., 83

Kumon, M., 21

L

Laggner, P., 02

Lawrence, S., 36

Lawrence, S.M., 37

Lee, W.-H., 61

Lewis, D., 35, 38, 64, 84

Lewis, D.A., 10

Lexmond, A.J., 50, 74

Littringer, E.M., 05, 62 
Llusa, M., 32, 36

Long, E., 84

Loo, C.-Y., 61

Lopes, I.S., 04, 08, 11, 70

Loughlin, R.M., 28

Ludlow, M., 29

Luinstra, M., 45

\section{M}

MacLoughlin, R., 52

Maia, F.M., 03, 04

Malamatari, M., 58

Markl, D., 37

Martin, A., 57

Martinelli, F., 83

Mason, F., 10, 35, 38, 64

Meindl, C., 55, 60

Mercuri, A., 12, 55, 60

Minami, H., 21

Misra, A., 49

Mitchell, J., 20

Mitchell, J.P., 68

Mohamed, S., 13

Mohr, S., 55, 60

Molokhia, A.M., 33

Mönckedieck, M., 82

Morris, C., 85

Moura, C., 25

Muddle, J., 54

Mukeria, E., 52

Munro, S., 15

Murnane, D., 18, 41, 57

Myatt, B., 35, 38, 84

\section{N}

Nagel, M., 20

Neves, F., 03, 04, 08, 11, 25, 70

Newcomb, A., 63

Newton, R., 84

Niven, R., 77

\section{O}

O'Shea, H., 35, 38

O'Sullivan, A., 28, 52

Offerhaus, H.L., 72

Oliveira, R.F., 07

Oliver, B., 34

Osman, N., 13

\section{$\mathbf{P}$}

Page, C., 54

Page, C.P., 50, 74

Palha, M., 04, 11
Pandya, S., 49

Parker, J., 48, 53

Parry, M., 14, 79

Patel, A., 24

Paudel, A., 02, 30, 31, 32, 36, 37, 47

Piazzoni, E., 59

Pinto, J.T., 47

Pope, F.D., 80

Pratt, B., 76

Price, R., 46

Prime, D., 65

\section{$\mathbf{R}$}

Rajoli, R.K.R., 09

Raula, J., 17

Renner, N., 42

Rhein, N., 06

Richter, A., 24

Ritchie, K., 13

Roberts, D.L., 68

Roblegg, E., 47

S

Sadler, R., 66

Sadler, R.C., 27

Salar-Behzadi, S., 12, 55, 60

Saleem, I., 13

Saleem, I.Y., 09

Sanders, M., 23, 26

Sandham, D., 85

Sarrailh, S., 59

Scalia, S., 61

Scherließ, R., 05, 06, 42, 82

Schiaretti, F., 55

Schneider, H., 20

Schroettner, H., 31

Schwarz, E., 05, 62

Sefton, S., 15

Selg, E., 51

Serôdio, P., 70

Seville, P.C., 80

Seyfang, K., 16

Shalash, A.O., 33

Shur, J., 46

Silva, S., 11

Simkova, K., 56

Slowey, A., 39

Smith, N., 85

Solomon, D., 14

Somavarapu, S., 58

Stalder, J., 56

Stebler, F., 56

Steckel, H., 05, 42, 62, 82

Stevens, N., 65
Stolnik-Trenkic, S., 27

Stranzinger, S., 55, 60

Suggett, J., 20

Sweeney, L., 28, 52

Symons, D., 18

T

Tanimoto, M., 21

Teale, P., 29

Teixeira, J.C.F., 07

Teixeira, S.F.C.F., 07

Todorova, Z., 42

Tong, H.-J., 80

Traini, D., 34, 61, 81

Tran, C., 26

Trotta, V., 61

Tweedie, A., 10, 64

U

Urbanetz, N., 82

V

van der Werf, T.S., 71

Vartiainen, V., 17

Versteeg, H., 84

Versteeg, H.K., 35, 38, 68

W

Wahl, V., 02, 30, 31, 37

Wake, A., 14

Walzel, P., 82

Ward, A., 80

Ward, D., 14

Wauthoz, N., 22

Western, K., 15

Williams, G., 59

Wittmann, R., 62

Wolf, A., 16

Wolf, G., 67

Woods, A., 24

$\mathrm{Wu}$, S., 12, 55, 60

Y

Yada, S., 21

Yilmaz, I., 50

Young, P., 34

Young, P.M., 61, 81

$\mathbf{Z}$

Zambelli, E., 55

Zellnitz, S., 12, 30, 31, 47

Zhao, P., 68

Zimmer, A., 02 